\title{
A New Palm-Print Image Feature Extraction Method Based on Wavelet Transform and Principal Component Analysis
}

\author{
Jia wei Li and Ming Sun* \\ China Agriculture University, \\ Beijing China \\ jjlijiawei@163.com
}

\begin{abstract}
In recent years, as one of the biometric identification technology, palm-print identification has received many reseachers' attention. To solve the key problem of palm-print recognition -- feature extraction, we propose a new method, which based on wavelet transform and principal component analysis. In general, we use wavelet transform to deal with palm print images and extract high-dimensional wavelet energy features, then reduce the dimensionality of high-dimensional wavelet energy features through principal component analysis, and remain the original feature energy maximally. The features extracted by this method not only reflect palm-print images' information maximally, but also achieve the goal of data dimensionality reduction. Experiments show, the correct recognition rates of new method are much higher than those traditional methods such as LDA [1], PCA [2], 2DPCA [3], ICA [4] and so on.
\end{abstract}

Keywords: Palm-print Recognition, feature extraction, wavelet transform, Principal component analysis.

\section{Introduction}

Recently, researchers have done much research on palm-print recognition technology, and proposed many methods of palm-print feature extractions. In these methods, there are mainly methods which based on the structure of feature extraction, such as pointfeature and line-feature; the time-frequency [5] analysis of feature extraction, such as fourier transform, wavelet transform [6,7]; the subspace method of feature extraction, such as PCA,ICA. However, the method effect of feature extraction which based on the point-feature and line-feature of palm-print will be unsatisfied in the situation of low resolution of palm-print images. Similarly, the feature extraction which based on Fourier transform gets high dimensional data which is not advantaged to the real time recognition algorithm. Therefore, we propose a new method which based on wavelet transform and PCA to extract features of palm-print more effective.

* Author for correspondence: drmingsun@163.com 


\section{Two-Dimensional Wavelet Transform}

Firstly, we use edge detect algorithm and corner detect algorithm to locate the ROI (region of interest) of palm-print .Figure 1 shows the original image, the edge of palm, the corner of palm edge and ROI of palm-print.

As we know, the palm-print image is a kind of image which is similar to the texture of cycle image, different regions of the ridge orientation and spatial frequency represent the inherent characteristics of palm-print, so we use 2-D wavelet transform to decompose the image which provide the spatial and frequency domain positioning properties and separate the energy characteristics of multi-resolution palm-print image. Let $I(x, y)$ denote the ROI of palm-print, according to equation (1) [8], transform the $I(x, y)$ by using 2-D wavelet.

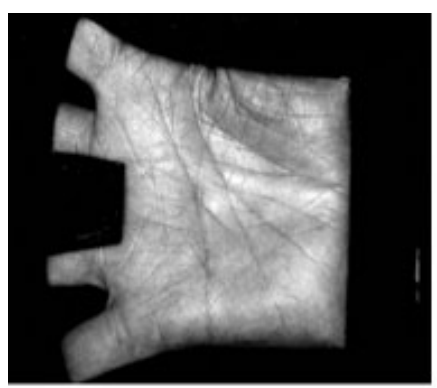

(a)

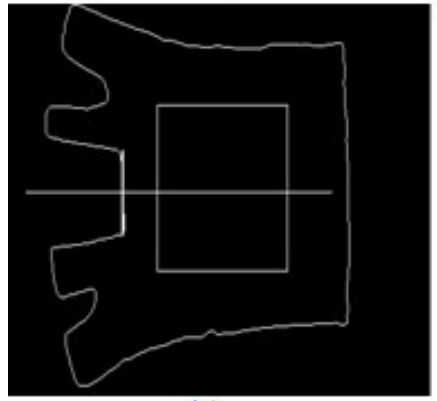

(c)

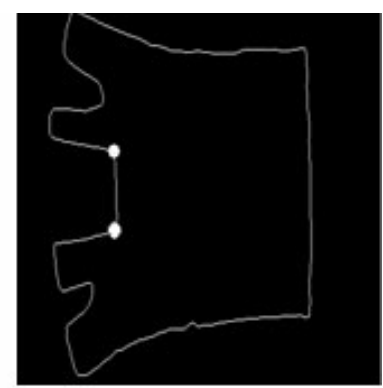

(b)

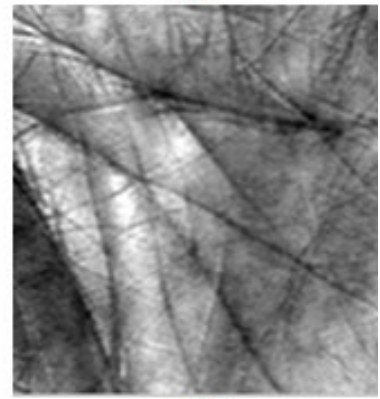

(d)

Fig. 1. An example of the preprocessing of palm-print (a) Original Palm-print (b) the edge and corners of palm-print(c) the chopped region of palm-print (d) Region of interest $(128 * 128$ pixels)

$$
w\left(a, b_{1}, b_{2}\right)=\frac{1}{a} \int_{-\infty}^{+\infty} \int_{-\infty}^{+\infty} I(x, y) \psi\left(\frac{x-b_{1}}{a}, \frac{y-b_{2}}{a}\right) d x d y
$$

Where $a$ is scale parameter, $b_{1}, b_{2}$ are translation parameters, $\psi(x, y)$ is 2-D wavelet transform functions. 
As the Haar wavelet have the characteristics of orthogonal, compactness and generalized linear phase, so in this paper, we use Haar wavelet to transform the ROI image, and decompose the image in three directions. Haar wavelet can be defined as:

$$
\psi_{H}(x, y)=\left\{\begin{array}{l}
1,0 \leq x<\frac{1}{2}, 0 \leq y<1 \\
-1, \frac{1}{2} \leq x<1,-1<y \leq 0 \\
0, \text { else }
\end{array}\right\}
$$

Decomposition algorithm [7] is as follows:

$$
\left\{\begin{array}{l}
d_{k}^{0}(l, i)=\sum_{y} \sum_{x} d_{k+1}^{0}(x, y) h(x-2 l) h(y-2 i) \\
d_{k}^{1}(l, i)=\sum_{y} \sum_{x} d_{k+1}^{1}(x, y) g(x-2 l) h(y-2 i) \\
d_{k}^{2}(l, i)=\sum_{y} \sum_{x} d_{k+1}^{2}(x, y) h(x-2 l) g(y-2 i) \\
d_{k}^{3}(l, i)=\sum_{y} \sum_{x} d_{k+1}^{3}(x, y) g(x-2 l) g(y-2 i)
\end{array}\right\}
$$

The result of wavelet transformed palm-print image is shown in figure 2 .

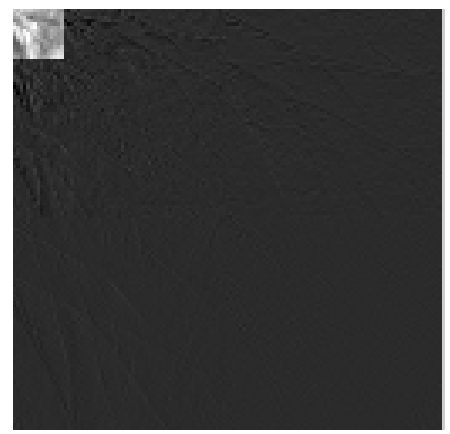

Fig. 2. An example of three level wavelet decomposition of palm-print image

Then we construct the wavelet energy feature of the decomposed image, $H_{i} V_{i}$ and $D_{i}$ are the $i$ level wavelet decomposition in the horizontal direction, vertical and diagonal directions of the details of the image.

The $i$ level wavelet energy in the corresponding directions of the image can be defined as $[9,10]$ :

$$
\begin{aligned}
E_{i}{ }^{h} & =\sum_{x=1}^{M} \sum_{y=1}^{N}\left[H_{i}(x, y)\right]^{2} \\
E_{i}{ }^{v} & =\sum_{x=1}^{M} \sum_{y=1}^{N}\left[V_{i}(x, y)\right]^{2} \\
E_{i}{ }^{d} & =\sum_{x=1}^{M} \sum_{y=1}^{N}\left[D_{i}(x, y)\right]^{2}
\end{aligned}
$$


These energy features reflect the edge intensity of the $i$ level decomposed wavelet image in horizontal, vertical and diagonal directions. The wavelet decomposition level coefficients on each component of the vector of energy are defined as follows:

$$
v_{i}=\left(E_{i}^{h}, E_{i}^{v}, E_{i}^{d}\right)_{i, 2, \cdots, M}
$$

Where $M$ is the number of the whole level of wavelet decomposition.

Obviously, the energy vectors $v_{i}$ reflect global feature of the palm-print image, and can not describe the information of different regions of the palm. To solve this problem, we divide each detailed image into $S \times S$ disjoint blocks, and calculate the wavelet energy of every sub-image. Finally, we use the energy vector of every block to construct multi-resolution texture information of palm-print. Palm-print texture feature vector can be defined as $\tilde{v}$ :

$$
\tilde{v}=\left(\tilde{v}_{(1)}^{1}, \tilde{v}_{(2)}^{1} \cdots, \tilde{v}_{(3 \times S \times S)}^{1}, \cdots, \tilde{v}_{(1)}^{\sim_{(1)}}, \tilde{v}_{(2)}^{M}, \cdots, \tilde{v}_{(3 \times S \times S)}^{M}\right)
$$

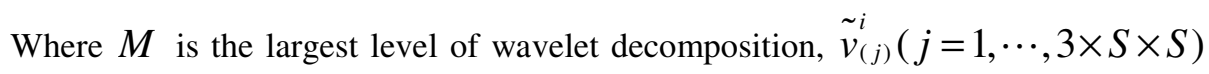
describes the energy of every block of three detailed images $\left(H_{i}, V_{i}, D_{i}\right)$, which is decomposed by wavelet.

Finally, we normalize $\tilde{v}$ as follows:

$$
\begin{aligned}
V & =\left(V_{(1)}^{1}, V_{(2)}^{1}, \cdots, V_{(1)}^{M}, V_{(2)}^{M}, \cdots, V_{\left(3 * S^{*} S\right)}^{M}\right) \\
V_{(j)}^{i} & =\frac{\tilde{v}_{(j)}^{i}}{\sum_{k=1}^{M} \sum_{l=1}^{3^{*} S^{* S}} \tilde{v}_{(1)}^{k}}(i=1, \cdots, M ; j=1, \cdots, 3 * S * S)
\end{aligned}
$$

According to equation (10), we define the $i$ level wavelet energy feature as follows:

$$
V^{i}=\left(V_{(1)}^{i}, V_{(2)}^{i}, \cdots, V_{\left(3 * S^{*} S\right)}^{i}\right)
$$

The $i$ level wavelet energy feature which reflects palm-print image in the different scale, location, and different texture features in different directions is calculated by the $i$ level wavelet coefficients. Then we get energy vector by construct every level wavelet energy feature as follows:

$$
V=\left(V^{1}, V^{2}, \cdots, V^{M}\right)
$$

The wavelet feature which composed by every level of energy wavelet reflects texture feature of palm-print image at different resolutions, different regions and different directions. 


\section{Principal Component Analysis}

As wavelet feature vector $V$ is high dimensional, so we use principal component analysis to deal with high dimensional features. The key is to find the PCA transform axis. And let's introduce PCA briefly. Suppose the variable $x$ has $N$ samples, $x_{k} \in R^{n \times 1}(k=1,2, \cdots, N)$, We define its mean vectors as $u$, then covariance matrix can be defined as follows:

$$
C=\frac{1}{N} \sum_{k=1}^{N}\left(x_{k}-u\right)\left(x_{k}-u\right)^{T} \in R^{n \times n}
$$

Here we define

$$
Z=\left[x_{1}-u, x_{2}-u, \cdots, x_{N}-u\right] \in R^{n \times n}
$$

Then we can get

$$
C=\frac{1}{N} Z Z^{T}
$$

We assume $W_{p c a}=\left[w_{1}, w_{2}, \cdots, w_{n}\right], w_{1}, w_{2}, \cdots, w_{n}$ as the eigenvector of $C$, then we can choose the number of dimensions of transformed data we want, if we set the dimension $r$, then we get $r$ eigenvectors $\left(w_{1}, w_{2}, \cdots, w_{r}\right)$ which correspond $r$ eigenvalues.

In this paper, we reduce the dimensions of the training samples' feature vectors $V$ by using PCA transform, and get $W_{v p c a}=\left[w_{v 1}, w_{v 2}, \cdots, w_{v r}\right]$ in PCA feature space.

The training samples' feature vectors $V$ are mapped to feature space, eigenvalues of $W_{v p c a}$ can be defined as follows:

$$
a_{v p c a}=\left[a_{v 1}, a_{v 2}, \cdots a_{v r}\right] .
$$

We use PCA to deal with every level wavelet energy features, and get reduced dimensional $V_{p c a}{ }^{i}=\left(V_{p c a}^{1}, V_{p c a}^{2}, \cdots, V_{p c a}^{M}\right)$, we call $V_{p c a}{ }^{i}$ as $P W E F$.

\section{Experiments and Results Analysis}

We test this feature extraction method on Polyu-Online-Plam-print Database. In the experiment ,we use 200 persons palm-print images, and choose 9 palm images of every person randomly .To every palm, we use 6 images as training set ,other 3 images as testing set. We use Haar wavelet to deal with these images samples, and get 5 level wavelet decomposed energy blocks ,every block is divided into $4 * 4$ subblocks, the vector $V$ 's dimension is 240 ,and then use PCA to deal with every block, 
finally ,we get every level's $P W E F$, the dimension of $P W E F$ is 40 . We use the mean value of every sample's $P W E F$ as the corresponding person's template.

In order to investigate the performance of the proposed approach, each sample in the database is matched against the other samples. The matching between palm-prints which are captured from the same palm is defined as a genuine matching. Otherwise, the matching is defined as an impostor matching. A total of 120,000 matching have been performed, in which 600 matching are genuine matching. We do this operation 5 times by using 5 levels $P W E F$. Figure 3 shows the genuine and impostor matching scores distribution. The scores of the genuine match of the first ,second ,third , forth, fifth level PWEF corresponding concentrates at 15,38,26,23,20, the scores of the impostor match of the first ,second ,third , forth, fifth level PWEF corresponding concentrates at 35,50,58,60,70. These two peaks are clearly separated and the distribution curve of the genuine matching scores intersects very little with that of impostor matching scores. Therefore, the proposed approach can very effectively discriminate between palm-prints.
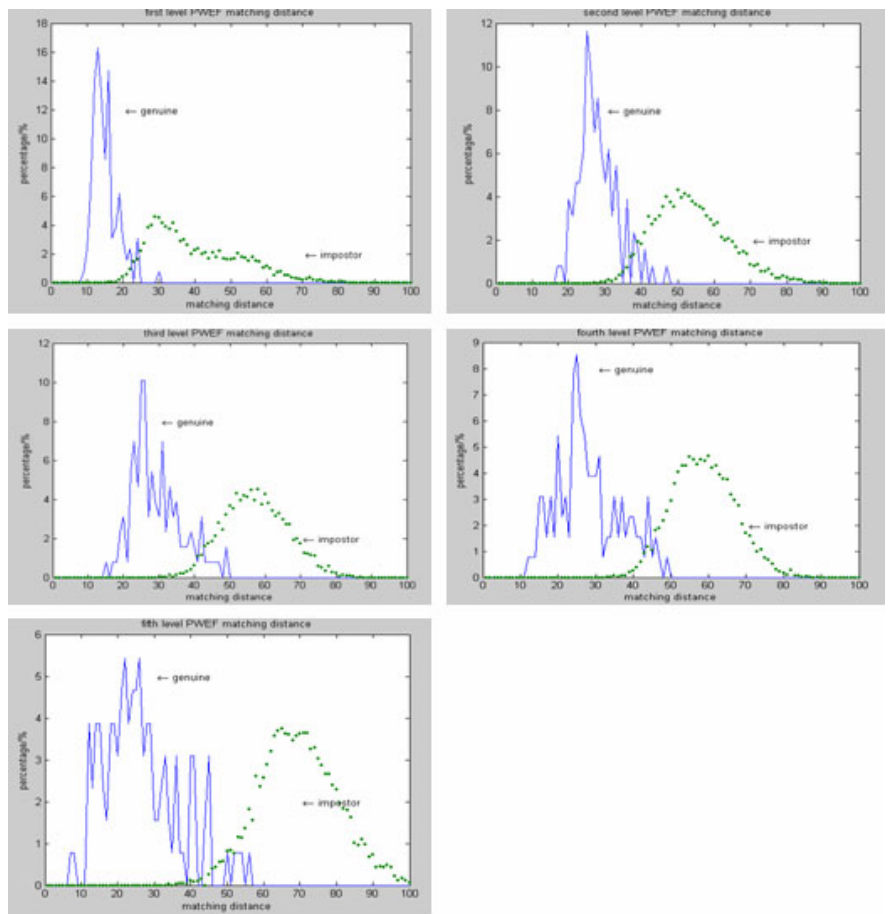

Fig. 3. The Five Levels $P W E F$ Distributions of Genuine and Impostor Matching Scores

In the processing of recognition, we use the nearest neighbor classifier to calculate the distance between testing sample and every training sample. The result of recognition is shown in table 1 . 
Table 1. Correct Recognition Rate, Training and Recognition Time

\begin{tabular}{|c|c|c|c|}
\hline Method & $\begin{array}{c}\text { correct } \\
\text { recognition } \\
\text { rate }\end{array}$ & $\begin{array}{c}\text { Training } \\
\text { time }\end{array}$ & $\begin{array}{c}\text { recognition } \\
\text { time }\end{array}$ \\
\hline PCA & $93.2 \%$ & $18.4313 \mathrm{~s}$ & $0.1094 \mathrm{~s}$ \\
\hline 2DPCA & $92.26 \%$ & $17.2537 \mathrm{~s}$ & $0.1503 \mathrm{~s}$ \\
\hline LDA & $96.7 \%$ & $22.7625 \mathrm{~s}$ & $0.1950 \mathrm{~s}$ \\
\hline ICA & $95.75 \%$ & $15.1397 \mathrm{~s}$ & $0.1538 \mathrm{~s}$ \\
\hline PCA\&LDA & $97.8 \%$ & $22.7625 \mathrm{~s}$ & $0.2210 \mathrm{~s}$ \\
\hline 1 level PWEF & $98.45 \%$ & $13.2356 \mathrm{~s}$ & $0.1235 \mathrm{~s}$ \\
\hline 2 level PWEF & $95.35 \%$ & $12.9683 \mathrm{~s}$ & $0.1300 \mathrm{~s}$ \\
\hline 3 level PWEF & $94.57 \%$ & $13.3987 \mathrm{~s}$ & $0.1247 \mathrm{~s}$ \\
\hline 4 level PWEF & $97.67 \%$ & $13.4735 \mathrm{~s}$ & $0.1267 \mathrm{~s}$ \\
\hline 5 level PWEF & $99.22 \%$ & $13.1848 \mathrm{~s}$ & $0.1295 \mathrm{~s}$ \\
\hline
\end{tabular}

We use the same training set to test PCA, 2DPCA, LDA, ICA, PCA \& LDA and find that recognition accuracy is improved by nearly three percentage points, the time of recognition improved $0.07 \mathrm{~s}$ averagely.

From the experiment of palm-print recognition, we get the ROC in figure 4 which reflects the performance of the proposed feature extraction algorithm.

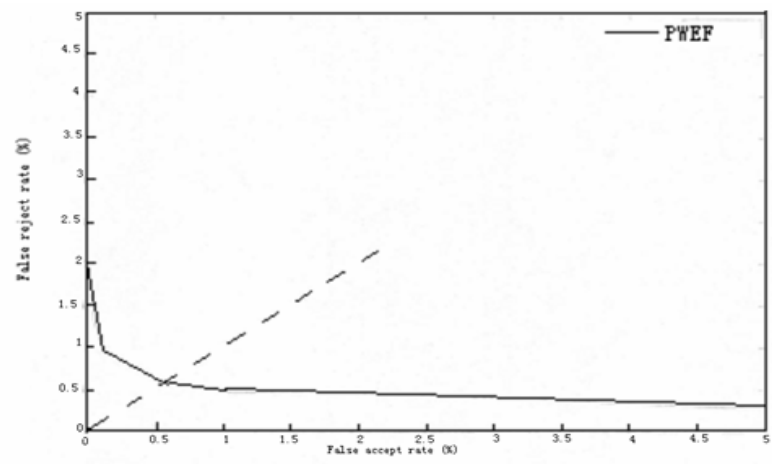

Fig. 4. The ROC Curve of the Proposed Approach

From the ROC Curve, we can see equal error rate reach about $0.60 \%$, so the recognition accuracy are able to meet our expected accuracy. And it fully illustrated PWEF feature extraction method in palm-print recognition system's advantage.

\section{Conclusion}

In this paper, we applied the combination of wavelet transform and PCA on palmprint image feature extraction. Firstly, we use edge detection algorithm and corner 
detection algorithm to locate the ROI of palm-print, secondly, we transform the ROI with Haar wavelet, thirdly, we use PCA to deal with the transformed ROI image and get the PWEF. Through the recognition experiment, the correct recognition rate increased nearly $3 \%$ and recognition time reduced about $0.07 \mathrm{~s}$. So the proposed method can describe palm-print texture features, and has a wide application in the field of biometric technology.

\section{References}

[1] Connie, T.J., Andrew, T.B.: An automated palm-print recognition system; Image and Vision computing, 501-515 (2005)

[2] Lu, G., Zhang, D., Wang, K.: Palm-print recognition using eigenpalms features. Pattern Recognition Letters 24(9210), 1463-1467 (2003)

[3] Li, Q., Qiu, Z., Sun, D., et al.: Online palm-print identification based on improved 2DPCA. Acta Electronica Sinica 33(10), 1886-1889 (2005)

[4] Wang, J., Zhang, Z., Zhang, Y., et al.: A guantitative analysis for complicated spectra based on independent component analysis. Journal of Optoelectronics 'Laser 18(6), 741745 (2007)

[5] Wu, X., Wang, K., Zhang, D.: A novel approach of palmline extraction. In: Proceedings of Third International Conference on Image and Graphics, pp. 230-233 (2004)

[6] Tico, M., Immonen, E., Ramo, P., et al.: Fingerprint recognition using wavelet features. In: Proceedings of 2001 IEEE International Symposium on Circuits and Systems, vol. 2, pp. 21-24 (2001)

[7] Mallat, S.: A Wavelet Tour of Signal Processing, 2nd edn. Academic Press, New York (1999)

[8] Mallet, S., Hwan, W.: Singularity detection and processing with wavelets. IEEE Transactions on Information Theory 38(2), 617-643 (1992)

[9] $\mathrm{Wu}, \mathrm{X}$. , Wang, K., Zhang, D.: Wavelet Energy Feature Extraction and Matching for Palm-print Recognition. Journal of Computer Science and Technology 20(5), 411-418 (2005)

[10] Wu, X., Wang, K., Zhang, D.: Wavelet based Palm-print Recognition. In: Proceedings of the International Conference on Machine Learning Cybernetics, pp. 1253-1257. IEEE, USA (2002) 